\title{
Patient perspectives of their leg ulcer journey
}

\begin{abstract}
- Objective: To understand the personal impact of venous leg ulceration from the patients' perspective. - Method: Face-to-face, unstructured interviews were conducted with nine patient participants with venous leg ulcers. The interviews were digitally recorded, transcribed verbatim and, using thematic analysis, the themes and subthemes which impacted on quality of life were identified.

- Results: Four core themes were identified: the ulcer, symptoms, wound management and effects on daily life, with 16 subthemes that negatively impacted on quality of life (QoL) also identified.

- Conclusion: This qualitative study offers a valuable insight into the complex issues that impact on daily living for this patient group. The implications of the findings are far reaching and suggest that proactive symptom management and the fostering of a patient focus to consultations may improve QoL and encourage the patient to engage as an active partner in his/her management plan; both of which are explored in the subsequent phases of the larger study.
\end{abstract}

- Declaration of interest: This study was funded by West Midlands Strategic Health Authority.The authors have no conflicts of interest to declare.

chronic venous leg ulcers; community nursing; interviews; quality of life; wound care

J. Green,' MSc, Lecturer and PhD Research Fellow; R. Jester, ${ }^{2,3} \mathrm{PhD}$,

Adjunct Professor and Specialist Project Lead; R. McKinley,' PhD, Professor of Education in General Practice, Keele Medical School; A. Pooler,' PhD, Lecturer and Post-Doctoral Research Fellow; I Keele University School of Nursing and Midwifery, Clinical Education Centre, University Hospital of North Staffordshire NHS Trust, Stoke-on-Trent, UK. 2 School of Nursing and Midwifery, Griffith University, Queensland, Australia.

3 Faculty of Education, HEFT, Birmingham: Email:j.green@ keele.ac.uk

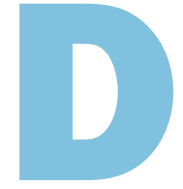

ue to the debilitating symptoms and the recalcitrant nature of the condition, people with chronic venous leg ulcers (VLUs) experience diminished quality of life (QoL). ${ }^{1-4}$ Over $40 \%$ of patients' ulcers last 12 months or more (median 6-9 months, range 4 weeks-72 years) and often recur $(26-69 \%),{ }^{1,5-9}$ with statistics improving little over the last 20 years. ${ }^{7,10}$ For some, ulcers last for much of their lifetime.

The chronicity of VLUs can be devastating, with effects on many areas of the life of the patient. ${ }^{11,12}$ Life is complicated by issues including pain, limited mobility, odour, depression and social isolation. ${ }^{1,13,14}$ Care is predominantly delivered in the community and is of varying quality, focusing primarily, if not solely, on the provision of wound care; often with little regard for the wider impact that ulceration poses..$^{1,4,12,15}$

This project aims to build on previous research and to establish those factors that impact on the daily lives of people with chronic VLUs. ${ }^{16,17}$

\section{Method}

A phenomenological design was employed to collect data via face-to-face, unstructured interviews. Such interviews allow participants to fully articulate their experiences and provide an excellent opportunity to understand behaviour. ${ }^{18}$

A two-stage sampling procedure was used. The first stage was of district nurse (DN) participants who cared for patients with VLUs and the second of patients with VLUs from their caseload. Nurses were recruited from two teams in two local primary care trusts (PCTs). Nurse participants were experienced in the care of patients with VLUs. All consenting nurse participants then purposively selected potential patient participants from their caseload, thus protecting confidentiality, and distributed study information and consent forms. The inclusion criteria for patients were chronic leg ulceration of venous or mixed aetiology for over 6 weeks and the ability to provide written informed consent.

Data were collected between June 2010 and January 2011; individual interviews were conducted, at times and locations convenient for the patients. Interviews were initiated using a single open-ended question ('What is your experience of leg ulceration?'), to invite the participants to reveal their experience of living with their VLU. Interviews were digitally recorded with the participants' permission and lasted between 30-120 minutes. Immediately after each interview, a reflective journal was completed to record observations about the interview and ideas about future coding. Interviews continued until data saturation was reached and no new themes were evident during analysis. ${ }^{19}$

Digital recordings were transcribed verbatim, and transcripts were checked for accuracy and analysed using thematic analysis..$^{20}$ This six-stage analysis

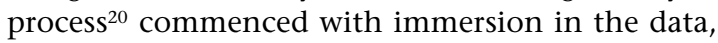
the logging of initial codes and more formal coding processes with themes formed. A thematic map of the data was created, providing the scope and content of themes. A final analysis of the data was then undertaken and written up to complete the process. This auditable process continued until no new themes were identified. 
Table I. Patient participant demographics

\begin{tabular}{|c|c|c|c|c|c|c|}
\hline $\begin{array}{l}\text { Participant } \\
\text { pseudonym }\end{array}$ & $\begin{array}{l}\text { Age } \\
\text { (years) }\end{array}$ & Gender & $\begin{array}{l}\text { Marital } \\
\text { status }\end{array}$ & Residential status & $\begin{array}{l}\text { Duration } \\
\text { of ulceration }\end{array}$ & $\begin{array}{l}\text { No. of } \\
\text { episodes }\end{array}$ \\
\hline Tom & 76 & Male & Married & With wife, own adapted bungalow & 10 years & $2-3$ \\
\hline Mary & 72 & Female & Married & With husband, own adapted bungalow & 30 years & $>5$ \\
\hline Evan & 76 & Male & Single & Private residential home & 35 years & $>5$ \\
\hline May & 99 & Female & Widow & Private residential home & 3 years & I \\
\hline Pam & 78 & Female & Married & With husband, own house & 30 years & 3 \\
\hline Ellen & 80 & Female & Widow & Private residential home & 2 years & I \\
\hline Steve & 39 & Male & Single & Alone, upstairs local authority flat & 14 years & I \\
\hline Margaret & 72 & Female & Widow & With dog, upstairs local authority flat & 20 years & $>5$ \\
\hline Sam & 86 & Male & Married & With wife, own house & 40 years & $>5$ \\
\hline
\end{tabular}

When a qualitative approach to research is employed, the data are not purported to be generalisable to the wider population. However, the internal validity of the study remains important. Veracity and auditability are vital, ${ }^{21}$ and these were enhanced by an ongoing reflexive approach to the research process. Consistency of both the collection of data and the analysis process was assured by a single researcher conducting all of the interviews and transcription. Braun and Clarke's structured framework ${ }^{20}$ was systematically applied to the interview data and, to confirm the accuracy of the analysis process and to optimise rigour, data from the interviews were also coded independently by an educational supervisor, ensuring transparency of the process.

Ethical approval for the study was granted by MidStaffordshire Local Research Ethics Committee. All participants received clear, written information about the study and their involvement. Written consent was gained prior to commencement.

\section{Results}

\section{Participant demographics}

The 13 nurse participants had worked in primary care for a median of 5 years (range 6 months to $>20$ years). Nine patients took part in the study; four were male (44\%), two lived alone, four with a partner and three lived in residential care. Patient participants had a median age of 76 years (range 39-99 years; Table 1 ).

\section{Themes and subthemes}

Four core themes were identified from the analysis of the interview transcripts: the ulcer, symptoms, wound management and the effects on daily life, with each theme encompassing a number of subthemes (Fig 1).

Each core theme, and the respective subthemes, are summarised and illustrated with verbatim quotations from the interview transcripts below. As strong local dialect was evident in many of the quotations, where necessary, meaning is explained in parenthesise. Participants are identified by pseudonyms.

\section{Theme I: the ulcer}

All participants were keen to describe their 'ulcer journey' and used the interview as an opportunity to outline the story of their ulceration. Reflections included their family history, any comorbidities and details about the ulcers, such as the cause, position and duration of ulceration.

Three participants' family history of VLUs were significant. Where such a history was present, participants seemed to be almost resigned to their apparent 'susceptibility' to VLUs.

'My sister has ulcers as well. All my mother's sisters had it, and my mother-runs in my family it does with us.' Tom

\section{'My Mum had them and they've told me they can be hereditary.' Margaret}

Comorbidities were common; three participants had no comorbidities, three had one and the remaining had two or more, which included rheumatoid arthritis $(n=2)$, osteoarthritis $(n=3)$, cardiovascular disease $(n=1)$ and sight problems $(n=2)$. Participants who suffered from comorbidities reflected that these were often exacerbated by problems related to their ulceration; for others, the ulcer was merely a minor irritation compared with the impact of other conditions.

\section{'The ulcers are a damn nuisance.' Tom}

Participants all spoke about the cause and the time span of their ulceration. For some a cause was clear; for others, their ulcer had simply appeared without warning. Others considered whether occupational factors had predisposed to their development of ulcers.

'Well, I've always had a job standing. Whether that has caused it...' Sam 
Some reflected on their self-management of the initial ulcer, before eventually having to accept that professional management was required. Often expert advice had become essential due to wound deterioration or the presence of infection. Others spoke of periods of avoiding professional management later in the course of their ulceration and of becoming non-concordant when the situation became too much for them:

'You just go through mad stages. Just trying not go, just thinking "I've done it." I'd phone and say "Sister, I don't need to come today, me bandages haven't leaked through." "Are you sure cause we can change them or come to you?" I'd say "No, you're alright, they haven't leaked through or nothing", but I'd done it myself. It was just a stage I went through with them; just trying not to have to go-three times a week-I mean, come on-it's tedious isn't it? They put them on on Monday, you go up Monday afternoon, you've got Tuesday all day and I'm back there on Wednesday; so it's only a day and a half they're staying on and then they're being changed.' Steve

Most had experienced some healed episodes $(n=7)$, although not all. For one participant in particular, it was taking years.

'This Christmas it'll be just over about 14 years. It's just been millimetres-millimetres all the time just going in very, very slow, cause I've not been anywhere, not done nothing for 12, 13, 14 years. All me life's been is... is doctors and hospitals and nurses and surgeons-you know, it does get to you [...] but I haven't let it get me down and I've stuck with it and yes, I'm doing alright now like, I'm getting there. It's getting there.' Steve

Lack of healing presented a significant challenge to some participants. Similarly, recurrence following an episode of healing was frustrating and disheartening.

'Off and on. I must have had them at least a dozen times.' Sam

'I think I've had about three or four, but the last two have been horrendous.' Margaret

The interviews revealed the personal narrative associated with leg ulceration. Comments were consistent and unprompted by all participants. These stories provided an insight into the person behind the ulceration, the extent of the impact of VLU on daily life and provided a background to their personal journey.

\section{Theme 2: symptoms}

All participants reported a range of debilitating symptoms due to their ulcers, providing three subthemes.
Fig I.Themes and subthemes identified from interviews

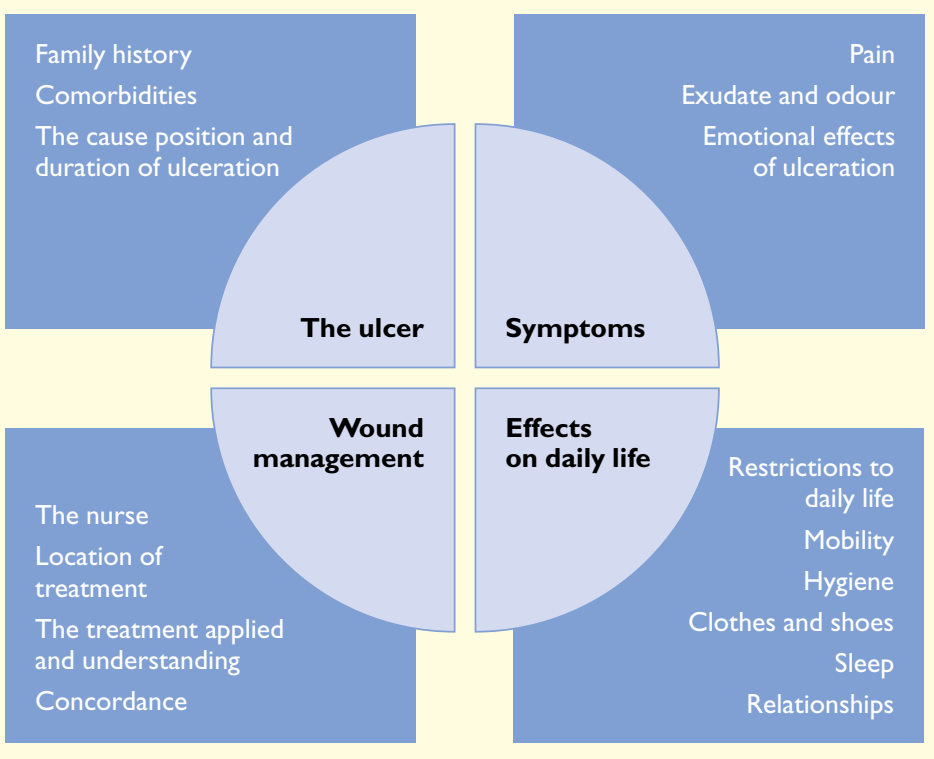

- Pain Pain dominated the lives of the participants and was the focus of every interview. Similarities in the descriptions of pain included its unceasing nature, and the severity and timing of the pain experienced, especially throughout the night.

'It was getting more painful, it was like one time it was like burning pain, then it was more like a stabbing pain, then. Now it's real sore, like someone is just rubbing, rubbing, rubbing, all the time. Oh, the pain-it's just unbearable.' Steve

'All through the night [...] you just can't get any rest.' Mary

'I have never had so much pain and they've made me feel so ill.' Margaret

One participant reflected:

'It's just the same pain, 24/7. I just have put up with it.' Steve

For all participants, there was a reluctance to take analgesia, often due to a cocktail of medication taken for their comorbidities.

'With all that I take for my arthritis, I figured it was covered.' Tom

Where analgesia was taken, it was felt by many to be ineffective for the type and intensity of pain experienced. 
'[...] but I do take the paracetamol-they're not brilliant, you know.' Margaret

'I don't take them unless I have to. I'd rather not take painkillers.' Sam

Pain was a central issue in every interview and was vividly described in terms of its severity and incapacitating nature.

- Exudate and odour The impact of exudate and odour was powerfully described by respondents and included reflections on the challenges that these issues posed to daily lives. These devastating symptoms were present for many of the participants and caused embarrassment, shame and stress.

'Oh, and when you first have them, I wondered what the smell was-it's terrible the smell, it all comes out, a lot of rubbish. When you went anywhere, you didn't get too close to people, because I can smell it, terrible.' Ellen

'It was a really offensive smell-you know what I mean, like it was like rotting flesh, it was horrible and, I smelled worse than a fishmongers, you know?' Steve

For some participants, in order to control the devastating effects of odour and exudate on their lives, they limited their contact with others and created a self-imposed isolation. This was seen as preferable to the embarrassment the symptoms caused.

'Social life? 'Err, I haven't got one. I just don't bother cause I know I've got to get myself better' Steve

'They stop you from going anywhere really. You know, you can't get about, not the same.' Ellen

- Emotional effects of ulceration Participants reported a range of emotional effects due to their ulceration, including depression, poor self-image and a fear of people's reactions. The interviews revealed a range of coping strategies adopted by participants. Some were striving to maintain their 'normal' functioning, whereas others suffered from anxiety and depression, with one respondent disclosing that he had had suicidal thoughts.

'It's just depressing really, if you think about it. I am on antidepressants. I just have to put up with itit's either that or kill myself.' Steve

'Terrible. Really down.' Evan

In contrast, another participant stated that, despite the profound impact of her ulceration, she endeavoured to continue her activities as before:
'It feels... I don't cry, but I could cry.' Margaret

Despite this, she went on to say:

'I tell you-you have to shake yourself. You have to shake your feathers and when you go out you have to put your outside face on. You know, you just have to.' Margaret

Despite the negative psychological impact of long-standing VLUs, the theme of hope was evident in many of the interviews; even for Steve who had experienced 14 years of ulceration.

'Yeah, I'm doing alright now like, I'm getting there. It's getting there.' Steve

These three debilitating subthemes were common across all interviews and were described clearly by participants.

\section{Theme 3: wound management}

Participant discourse about their wound management revealed the importance they placed on the physical management of their wounds. The central involvement of health professionals in the leg ulcer journey was present in all of the interviews. A number of participants reflected on their preference for consistency in their nursing team;

'[...] with the consistency of a team, much better. They did once send another from another surgery out, it wasn't the same. When you're seeing someone only once, it isn't the same. Nothing wrong with her, did the job just the same-fine-but I wasn't used to her.' Tom

Similarly, other participants said:

'It would be better if you saw the same nurse really, cause they would get to know what it's like.' Sam

'[...] you'd go and there would be girls there and you'd perhaps see them twice and then you wouldn't see them again. So, you'd get somebody else, so somebody else has a different way of doing it; so you didn't know where you were.' Margaret

For many, their relationship with the nurse was special; some were seen as friends, with close bonds made over the course of many visits.

\section{'[...] had some lovely nurses-they've been brilliant.' Tom}

Some participants attended clinics for their wound management, while others were seen at home. Some reflected on the time wasted waiting for nurses to 
visit. One gentleman in a residential home reflected on missing activities while waiting for his visit.

\section{'Sometimes... sometimes when the nurses were late I'd have to wait.' Evan}

The decision to attend clinic had been a conscious one for some participants, in order to be in control.

'You know, when I first went they said which would you prefer-do you want to come here or do you want us to come to your house? And I just said that I'd come up to clinic. I just thought moving about a bit would be better, might do me better than just sitting about.' Sam

One participant reflected on difficulties travelling home from clinic following their dressing change:

'They have a clinic down at our doctors, on three days a week for dressings. I'll go to it, but sometimes, you know, sometimes I have a job to come home when it's just been dressed.' Mary

All participants mentioned the wide variety of wound-care products that had been used on their ulcers over time. Many demonstrated excellent knowledge about the products on offer. One stated:

'You name it, all the different patches with stuff in and creams and the patches come out with the silver in and we went through every one of them. Errr... I've gone through loads of different stuff-they've put, I've had trials of different stuffs put on and some worked and some hasn't.' Steve

'Yes, they tried all sorts, you know. I think I've had iodine and different sorts of things.' Ellen

In contrast, one participant reflected on a difficult relationship with her nurses due, she felt, to their perception of her as being non-concordant. This participant felt she had been reprimanded by the nurses on a number of occasions for removing her dressing in between clinic visits and reflected:

'As I say, they're [nurses] great with me on the whole, but then they started getting cross that I washed me feet at night... er, apart from showering I do wash me feet at night before I go to bed anyway, and they got a bit cross.' Pam

The relationship with the nurse was extremely important to all participants. The interviews demonstrated a distinct focus on wound healing as the goal of treatment, with many participants reflecting that this was often elusive and, when achieved, difficult to maintain.
'I think I've had about three or four, but the last two have been horrendous.' Margaret

'I've had them twice this year.' Sam

\section{Theme 4: effects on daily life}

A number of subthemes referred to the effects of ulceration on the participant's daily life.

- Restrictions to daily life Some participants reflected that they stayed at home because the activities usually undertaken each day had become more difficult. Others stayed at home in order to limit their contact with others or to avoid further injury. For whatever reason, normal daily life was interrupted for many as a result of ulceration.

'I'm frightened in the supermarket. I am frightened when I'm out, when I have been at the supermarket cause some people, they do push their trolleys everywhere. So it means that you're on your guard all the time.' Margaret

'Well, they stop you from going anywhere really. You know, you can't get about, not the same.' Ellen

Some felt they rose to this challenge and, with determination, went out despite their ulceration.

\section{'I don't let anything restrict my life.' Pam}

This, for some, was their attempts to almost fight back against the limitations their ulcer imposed, in order to maintain normal functioning.

- Mobility Many respondents reported difficulties with walking, either due to discomfort from their wound or due to the dressings. Many also had a fear of falling.

'I can't walk. Yes, you walk but I'm frightened, because I put my foot out, you're frightened of falling.' Margaret

- Personal hygiene The maintenance of personal hygiene was extremely important, but difficulties in this area were raised by most participants. Discussion focused on problems bathing or showering due to the dressing. Some had used a new leg appliance that had improved functioning in this area. Others spoke of the need to have their legs washed between dressing applications. One lady talked about the dressing procedure undertaken by her nurses', stating that her legs were not washed, which upset her:

'No, they cut it all off and then she just puts a bit of cream on and that's it. Another bandage on.' Ellen

- Limited choices for clothes and shoes All participants raised issues with restricted choices for 
clothes and shoes. Problems were most often attributed to the bulkiness of the dressings required, which made choices of shoes difficult. Others felt a need to conceal their dressings.

'It's horrible-you can't dress as you want to. I've got nice fine skirts as I could have, you know, printed skirts for the summer, ever so nice.' Margaret

\section{'I can't get my shoes on.' Evan}

'I have to undo the laces and untie'em [them] like, so if I do go for a pair of trainers like, it's hard-I have to get a pair a size bigger, cause of all the bandages. I mean I take $9 \mathrm{~s}$, these are $10 \mathrm{~s}$ and I went to buy a pair the other day and they were 11s, and they just looked like that and I thought I can't wear them, they look like boats.' Steve

- Sleep was an issue for all respondents, most often due to pain. One participant commented that night times were particularly difficult:

'It is-when you've been in bed, it wakes you up.' Ellen

'Some nights, err... I had no sleep with it all night-it was going like this [indicates clenching motion] every few minutes, and you're there trying to find somewhere to put your leg, you know. It's awful.' Margaret

The lack of sleep accentuated the debilitating nature of the condition and made day-to-day functioning more difficult.

- Relationships A number of the interview participants reflected on the effect of their ulceration on their relationships. Some required family members to assist them with daily activities and, in some cases, carers had become the cared for. One participant sadly reflected on his inability to provide the level of care for his wife that he usually did:

'I haven't been able to go round the supermarket I just haven't been able to manage it-I'd have to sit down and my wife would struggle round.' Sam

The youngest participant reflected on the effects of his ulcers on forming intimate relationships. He reflected:

'[...] but relationship wise, er no chance. I couldn't, once these have healed then, obviously yes, but it's just, you know, with these on me legs all the timethere was one girl and I tried, she said "what's all that on yer [your] legs?" and I tried make out that I'd been in a fire and I'd burned meself [myself], but with the smelling and that, it dinna [didn't] last and I've just sort of put it off.' Steve
Day-to-day living for all participants was a challenge, with leg ulceration impacting on every aspect of their lives.

\section{Discussion}

The findings of this study clearly establish the pervasive and profound effect of VLUs on the daily lives of the patient. The impact on physical, psychological and social functioning and QoL is overwhelming for many of these participants. Participants demonstrated a range of responses to the impact of their ulcers; some participants saw it as a challenge and, despite their difficulties, did all they could to maintain usual functioning. In contrast, others withdrew from their normal activities, limiting their contact with others until their condition would improve.

This study reinforces and extends our understanding of the impact of VLUs. Pain overwhelmingly dominated the lives of participants and impacted on every aspect of their functioning. It interrupted sleep, limited mobility, lowered mood and proved difficult to manage. Pain was portrayed as 'continuous' and 'unbearable', a constant reminder of ulceration throughout a number of studies. ${ }^{2,11,12,22,23}$ Ebbeskog and Ekman ${ }^{24}$ reflected that pain was central to their participants' lives, making them 'cry in despair'. Difficulties controlling the pain have similarly been reported, with analgesia deemed to be ineffective against ulcer related pain. The enduring nature of the pain experienced and the inadequacy of the analgesic options available to these study participants are themes not disclosed as compellingly in earlier studies and serve to highlight the need for further research into this area, to successfully manage symptoms and meet the needs for effective pain relief for this patient group..$^{24,25}$

Difficulties managing wound exudate and odour were evident for all and had profound effects on every aspect of functioning, resulting in embarrassment, shame and social isolation. The sheer embarrassment of odour, its impact of self-image and the resultant self-imposed isolation as a way of preventing others from being exposed to this humiliating symptom were also key findings. Odour and leakage were similarly acknowledged in other studies, ${ }^{1,4,24,25}$ serving to highlight the need for more effective wound management strategies.

The composite effect of pain, exudate and odour was to severely limit social functioning and to lower mood, even prompting thoughts of suicide for one participant. Despite these serious limitations, others fought to maintain their functioning; attempting to engage as they had before their current episode of ulceration and a theme of hope, especially for healing, was evident for all. For many, where the healing process is prolonged and their ulcer recurrence almost inevitable, the focus of care on healing as the sole outcome of care has been questioned. Indeed,

\section{References}

I Briggs, M., Flemming, K. Living with leg ulceration: a synthesis of qualitative research. J Adv Nurs. 2007; 59: 319-328.

2 Chase, S., Melloni, M., Savage,A.A forever healing: The lived experience of venous ulcer disease. JVasc Nurs. 1997; XV: 73-78.

3 Franks, P., Moffatt, C. Health related quality of life in patients with venous ulceration: Use of the Nottingham health profile. Qual Life Res. 2001; 10: 693-700.

4 Persoon, A., Heinen, M. van derVleuten, $C$. et al. Leg ulcers: a review of their impact on daily life.J Clin Nurs. 2004; 13: 34 I-354. 5 Callam, M., Ruckly, C. Harper, D., Dale, J. Chronic ulceration of the leg: extent of the problem and provision of care. BMJ. 1985; 290: 1855-1856. 6 Moffatt, C., Franks, P., Oldroyd, M. et al. Community clinics for leg ulcers and impact on healing. BMJ. 1992; 305: 1389-1392.

7 Nelzen, O., Bergqvist, D., Lindhagen, $A$. long term prognosis for patients with chronic leg ulcers: a prospective study. Eur J Vasc Endovasc Surg. 1997; 13: 500-508

8 Nelson, E.A., Harper, D.R., Prescott, R.J. et al. Prevention of recurrence of venous ulceration: randomised controlled trial of class 3 and class 3 elastic compression. JVasc Surg. 2006; 44: 803-808.

9 Briggs, M., Closs, S.J.The prevalence of leg ulceration: a review of the literature. EWMA J. 2003; 3: 2, 14-20. 10 Heit, J., Rooke, T. Silverstein, M. et al. Trends in the incidence of venous stasis syndrome and venous ulcer:A 25-year populationbased study. JVasc Surg. 200 I; 33: 1022-1027. 
Briggs and Flemming ${ }^{1}$ recommend the adoption of the management of leg ulceration as a chronic condition; suggesting that the focus on healing may actually intensify the 'hopelessness' felt by the patient. They recommend that a renewed focus may improve coping strategies, serve to enhance the patient focus of consultations and encourage the nurse to move away from a focus solely on the wound. ${ }^{4}$

All participants reflected positively about the expertise and support offered by their nurses. Many commented on their preference for a consistent nurse, citing this as a key factor in improving the personal nature and the effectiveness of the care they received. Consistent care, competence in dressing application and regular feedback on wound progress was all considered key factors in the quality of the care delivered. Participants had experienced a wide variety of wound care products over time and were knowledgeable about their wound management strategy; often seeing themselves in partnership with their nurse against the ulceration. Other studies highlight similar positive effects of the nurse-patient relationship. ${ }^{24,26}$ For just one participant in this study the nurse-patient relationship had become difficult; a finding supported in some earlier studies where negative effects of the nurse-patient relationship were highlighted as often being the result of perceived non-concordance to treatment recommendations..$^{2,11}$

For many studies, as with this, the physical effects of VLUs dominated participant reflections. Daily living was a challenge for all. Getting out and about, mobility and maintaining personal hygiene were impaired due to the wound, the dressing or both; as were choices in what to wear. Sleep was regularly disturbed, most often by pain, and relationships were altered with carers becoming cared for and intimacy avoided. Participants were fearful of what people thought, of wounds deteriorating, of recurrence and, ultimately, of not healing. Ulceration affected every area of functioning over long periods of time. Other studies have similarly found that the dominance of the physical effects of ulceration may dilute the disclosure of psychological and social issues during

I I Hyde,C.,Ward, B., Horsfall, J., Winder, G. Older women's experience of living with chronic leg ulceration. Int J Nurs Pract. 1999; 5: 189-198.

I 2 Rich,A., McLachlan, L. How living with a leg ulcer affects people's daily life: a nurse-led study. JWound Care. 2003; I2: 51-54 13 Posnett, J., Franks, P.The costs of skin breakdown and ulceration in the UK. In: Skin Breakdown The Silent Epidemic. Smith \& Nephew Foundation, 2007. 14 Jones, J., Nelson, E.A. Skin grafting for venous leg ulcers Cochrane Database Syst Rev. 2005; I: CD001737.

interviews ${ }^{26}$ - this did not appear to be the case during this study. Participants spoke at length about the impact on their psychological functioning; describing the influence on their mood, motivation and engagement in activities. Often, when combined with the physical symptoms of ulceration, these two areas served to limit social functioning.

\section{Further research}

Reflections on the 'journey' of leg ulceration were common to all participants in this study; using the opportunity to speak unhindered to describe their experiences of healing and recurrence over time has also been described by Briggs and Flemming. ${ }^{1}$ Telling the story of the ulcer seems to be an important component of care for VLUs and is often overlooked in current care delivery. ${ }^{1,13}$ Fostering of a therapeutic relationship, where the patient feels valued, supported and listened to and where the chronicity of their ulceration is understood, is vital and, research claims, may result in improved healing rates and reduced ulcer recurrence. ${ }^{1,20}$ Research is needed to determine whether this is so.

\section{Conclusion}

This study suggests that the QoL of patients with VLUs is impaired in physical, social and psychological domains. The impact of ulceration is described together with the life-changing, debilitating symptoms, which can be unsuccessfully managed and have an enervating effect on all aspects of daily living. Understanding this 'lived experience', listening to the patient and providing effective symptom management for this chronic condition during consultations seems crucial to improving the QoL of this patient group. Further phases of this study will explore current consultations and pilot a tool designed to enhance patient centredness. The importance of the nurse-patient relationship and the need for consistency of care provision highlighted by this study is an area, in the light of these widespread NHS changes, that needs to be of central importance for decision makers.

19 Guest, G., Bunce,A., Johnson, L. How many interviews are enough? An experiment with data saturation and variability. Field Methods. 2006; 18: 59-82. 20 Braun,V., Clarke,V.Using thematic analysis in psychology. Quality Res Psychol. 2006; 3:77-10I. 2 I Lincoln, Y., Guba, E. Naturalistic Inquiry. Sage, 1985.

22 Walshe, C. Living with a venous leg ulcer: a descriptive study of patients' experiences. J Adv Nurs. 1995; 22: 1092-I 100. 23 Hopkins, A. Disrupted lives: investigating coping strategies for non-healing leg ulcers. $\mathrm{Br} J$ Nurs. 2004; 1 3: 556-563.
24 Ebbeskog, B., Ekman, S. Elderly people's experiences: the meaning of living with venous leg ulcer. EWMA J. 200 I; I: I, 2I-23.

25 Douglas, V. Living with a chronic leg ulcer: an insight into patients' experiences and feelings. JWound Care. 200I; 10: 355-360. 26 Brown, A. Leg ulcers. chronic leg ulcers, part 2: do they affect a patient's social life? $\mathrm{Br}$ ] Nurs. 2005; 14: 894-896. 